\title{
Ligustrazine inhibits platelet activation via suppression of the Akt pathway
}

\author{
LI LI $^{1,2^{*}}$, HONGWEI CHEN ${ }^{1,3^{*}}$, ALING SHEN ${ }^{1,3}$, QIONGYU LI $^{1,3}$, YOUQIN CHEN $^{4}$,

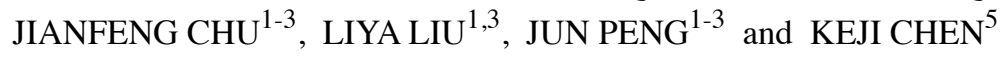 \\ ${ }^{1}$ Academy of Integrative Medicine, ${ }^{2}$ Chen Keji Academic Thought Inheritance Studio, \\ ${ }^{3}$ Fujian Key Laboratory of Integrative Medicine on Geriatrics, Fujian University of Traditional Chinese Medicine, \\ Fuzhou, Fujian 350122, P.R. China; ${ }^{4}$ Department of Pediatric Gastroenterology, Rainbow Babies and Children's Hospital, \\ Case Western Reserve University School of Medicine, Cleveland, OH 44106, USA; ${ }^{5}$ Department of Cardiology, \\ Xiyuan Hospital, China Academy of Chinese Medical Sciences, Beijing 100091, P.R. China
}

Received April 9, 2018; Accepted October 19, 2018

DOI: 10.3892/ijmm.2018.3970

\begin{abstract}
Aberrant activation of platelets has a critical role in thrombotic vascular events, including atherosclerosis, arterial thrombosis and myocardial infarction. The process of platelet activation is associated with multiple intracellular signaling pathways, including the phosphoinositide 3-kinase/AKT serine/threonine kinase (Akt) pathway. The well-known medicinal herb Rhizoma Ligusticum Wallichii (RLW) has long been used in China to clinically treat various cardiovascular disorders. As the most pharmacologically active component of RLW, ligustrazine has been demonstrated to possess a potent antiplatelet activity. However, the precise mechanisms mediating the bioactivities of ligustrazine have not been thoroughly elucidated. The present study evaluated the effects of ligustrazine hydrochloride ( $\mathrm{LH}$; the clinical-grade form of ligustrazine) on platelet activation and investigated the underlying molecular mechanisms. In vitro and ex vivo platelet activation models were used, established by stimulating rat platelet-rich plasma either with the platelet activator adenosine
\end{abstract}

Correspondence to: Professor Keji Chen, Department of Cardiology, Xiyuan Hospital, China Academy of Chinese Medical Sciences, 1 Xiyuan Caochang Road, Beijing 100091, P.R. China E-mail: kjchenvip@163.com

Dr Jun Peng, Academy of Integrative Medicine, Fujian University of Traditional Chinese Medicine, 1 Qiuyang Road, Fuzhou, Fujian 350122, P.R. China

E-mail: pjunlab@hotmail.com

${ }^{*}$ Contributed equally

Abbreviations: ADP, adenosine diphosphate; IGF-1, insulin-like growth factor-1; LH, ligustrazine hydrochloride; PRP, platelet-rich plasma; RLW, Rhizoma Ligusticum Wallichii; TXB2, thromboxane B2

Key words: LH, thrombosis, platelet activation, platelet aggregation, AKT serine/threonine kinase pathway diphosphate (ADP) or with the specific Akt pathway activator insulin-like growth factor-1 (IGF-1). The results demonstrated that treatment with LH significantly and dose-dependently inhibited ADP-induced platelet aggregation, in addition to thromboxane A2 (TXA2) secretion and intracellular $\mathrm{Ca}^{2+}$ mobilization in platelets, in vitro and ex vivo. In addition, LH markedly suppressed ADP-induced Akt phosphorylation in vitro and ex vivo. Furthermore, LH markedly inhibited IGF-1-induced Akt phosphorylation, platelet aggregation, TXA2 formation and $\mathrm{Ca}^{2+}$ mobilization in vitro. Finally, $\mathrm{LH}$ was able to reverse adrenaline-induced shortening of bleeding time. Taken together, these results suggested that ligustrazine possesses a broad range of antiplatelet activities without apparent hemorrhagic side-effects, and suppression of Akt signaling may be one of the mechanisms by which ligustrazine exerts its antiplatelet activities.

\section{Introduction}

Platelet activation is an essential process to repair injured blood vessels, restoring blood vascular integrity, which is fundamental for the maintenance of vascular function. However, aberrant platelet activation or hyperactivation of platelets may give rise to thrombosis and result in thrombotic vascular events, including atherosclerosis, arterial thrombosis and myocardial infarction (1-5). Under physiological conditions, platelets circulate through vessels with an intact and healthy endothelium, remaining in an inactivated state. However, when the endothelium is broken, the von Willebrand factor on the injured vascular wall interacts with its receptor located on the surface of platelets, which in turn induces platelet adhesion to extracellular matrix (6). Platelet activation results in the release of secondary mediators, including adenosine diphosphate (ADP), thromboxane A2 (TXA2) and thrombin (7). These molecules promote further adhesion, activation and aggregation of platelets, eventually forming a platelet plug to stop bleeding and to repair injured endothelium. The process of platelet activation is strongly associated with multiple intracellular signaling pathways, including the phosphoinositide 3-kinase (PI3K)/AKT serine/threonine 
kinase (Akt) pathway. Following activation by extracellular stimuli, PI3K is able to phosphorylate $\mathrm{PI}(4) \mathrm{P}$ and $\mathrm{PI}(4,5) \mathrm{P} 2$ to generate $\mathrm{PI}(3,4) \mathrm{P} 2$ and $\mathrm{PI}(3,4,5) \mathrm{P} 3$, respectively $(8)$. Akt is recruited by PIP3 to the platelet plasma membrane with the PIP3-binding domain, and phosphorylated/activated by 3-phosphoinositide dependent protein kinase 1 (PDPK1, additionally termed PDK1) and mammalian target of rapamycin complex 2 (mTORC2) (9-11). Activated Akt has critical roles in platelet function by mediating various cellular responses, including granule secretion, platelet aggregation and thrombus formation (12-15). Therefore, inhibition of platelet activation by suppressing the PI3K/Akt pathway may be a promising strategy to treat thrombotic vascular diseases.

A variety of antiplatelet drugs (including aspirin and clopidelgrel) demonstrate significant antiplatelet efficacy for treatment of thrombotic vascular diseases. However, the administration of most currently-used platelet activation inhibitors frequently results in bleeding complications and drug resistance $(16,17)$. Recently, an increasing number of studies are focusing on medicinal herbs, in order to discover complementary and alternative compounds with relatively fewer side-effects. Rhizoma Ligusticum Wallichii (RLW) is a well-known medicinal herb, which has long been used in China to clinically treat various cardiovascular disorders (18). Ligustrazine is one of the most pharmacologically active compounds of RLW, which has been used for anticardiovascular (19), antiplatelet (20), ischemic stroke (21), anti-Alzheimer's (22), neuroprotective (23) and anticancer (24) treatment. In cardiovascular and cerebrovascular diseases, ligustrazine exhibits significant therapeutic activity and may improve the microcirculation, by expanding small arteries, removing blood stasis, and by additionally having effects on antiplatelet aggregation, antioxidation, calcium antagonists and antifibrosis (18,25-29). However, the molecular mechanism of its mode of action has not been thoroughly elucidated. A preliminary study from our group identified that treatment with ligustrazine significantly inhibited ADP-induced platelet aggregation and Akt phosphorylation (Li et al, unpublished data). Therefore, it was hypothesized that ligustrazine hydrochloride (LH; the clinical-grade form of ligustrazine) may exhibit antiplatelet activities by suppressing the Akt signaling pathway. To confirm this hypothesis, the present study used in vitro and ex vivo platelet activation models, established by stimulating rat platelet-rich plasma (PRP) either with the platelet activator ADP or with the specific Akt pathway activator insulin-like growth factor-1 (IGF-1). The effects of $\mathrm{LH}$ on platelet activation and the underlying molecular mechanisms were investigated.

\section{Materials and methods}

Materials and reagents. Adrenaline hydrochloride was purchased from Fuyao Group (Fuzhou, China). A thromboxane B2 (TXB2) enzyme immunoassay kit was obtained from Enzo Life Sciences, Inc. (Farmingdale, NY, USA). Antibodies against phosphorylated (p-)Akt, Akt, $\beta$-actin, and horseradish peroxidase (HRP)-conjugated secondary antibodies were purchased from Cell Signaling Technology, Inc. (Danvers, MA, USA). ADP, fluo-4-acetoxymethyl ester (Fluo-4 AM), IGF-1 and other unstated chemicals were purchased from Sigma-Aldrich (Merck KGaA, Darmstadt, Germany).

Preparation of LH. LH (>99.0\%) was purchased from Beijing Putian Tongchuang biotechnology Co., Ltd. (Beijing, China). LH was dissolved in saline at a concentration of $20 \mathrm{mg} / \mathrm{ml}$ for ex vivo experiments or $180 \mathrm{mM}$ for in vitro experiments. The chemical structures of ligustrazine and LH are presented in Fig. 1.

Animals and treatment with LH. Male Sprague-Dawley (SD) rats of 6-8 weeks of age (200-250 g) were purchased from Shanghai SLAC Laboratory Animal Co., Ltd. (Shanghai, China). All animals were housed in a specific pathogen-free environment with food and water supplied ad libitum throughout the experiment. The environment was maintained at $22^{\circ} \mathrm{C}$ with a 12 -h light/dark cycle and humidity of $55 \pm 5 \%$. All the animal treatments were performed in compliance with international ethical guidelines and the National Institutes of Health Guidelines for the Care and Use of Laboratory Animals (Bethesda, MD, USA). The experiments were approved by the Institutional Animal Care and Use Committee of Fujian University of Traditional Chinese Medicine (Fuzhou, China).

For the ex vivo experiment, $24 \mathrm{SD}$ rats were randomly divided into the following three groups ( $\mathrm{n}=8 /$ group): Control, Adrenaline and Adrenaline + LH. The Adrenaline + LH group was administered LH $(80 \mathrm{mg} / \mathrm{kg}$; based on the clinical dosage and transformation between human and rat) by intraperitoneal injection daily for 7 days, while the other two groups received an equal volume of saline. At the end of the treatment, the rats in the Adrenaline $+\mathrm{LH}$ and Adrenaline groups were subcutaneously injected with adrenaline hydrochloride at a dose of $1 \mathrm{mg} / \mathrm{kg}$, whereas, the rats in the Control group were subcutaneously injected with an equal volume of saline. At $1 \mathrm{~h}$ following the injection, the bleeding risk of SD rats from each group was evaluated and the blood samples were collected for further ex vivo experiments (including assays of platelet aggregation, analysis of intracellular $\mathrm{Ca}^{2+}$ mobilization and TXB2 levels, in addition to expression of associated proteins).

Blood collection and preparation of rat platelets. Blood was collected from SD rats with or without LH treatment. Rats were anesthetized with sodium pentobarbital $(45 \mathrm{mg} / \mathrm{kg})$ and blood was collected from the abdominal aorta and anticoagulated with $3.8 \%$ sodium citrate solution $(9: 1, \mathrm{v} / \mathrm{v})$. The obtained blood samples (8-10 $\mathrm{ml}$ blood for each rat) were centrifuged at room temperature for $15 \mathrm{~min}$ at $150 \mathrm{x}$ g to obtain PRP, which were centrifuged again at room temperature for $15 \mathrm{~min}$ at $150 \mathrm{x} \mathrm{g}$ to remove residual erythrocytes. The platelet-poor plasma (PPP) was obtained by centrifugation of blood samples at room temperature for $10 \mathrm{~min}$ at $1,000 \mathrm{x}$. The final concentration of platelets was adjusted to $3 \times 10^{8} / \mathrm{ml}$ with the PPP, which was used as a reference solution for aggregation assays.

Assay of platelet aggregation. Platelet aggregation analysis was performed as previously described (21). For the in vitro experiment, subsequent to the preparation of PRP as aforementioned, $330 \mu 1$ rat PRP was incubated with 5.5 $\mu \mathrm{M}$ ADP or $300 \mu \mathrm{M}$ IGF-1 for $5 \mathrm{~min}$ at $37^{\circ} \mathrm{C}$, following pretreatment with 


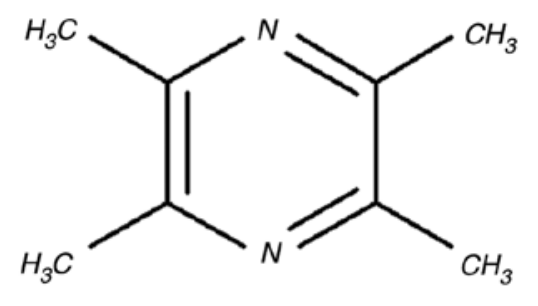

Chemical structure of ligustrazine

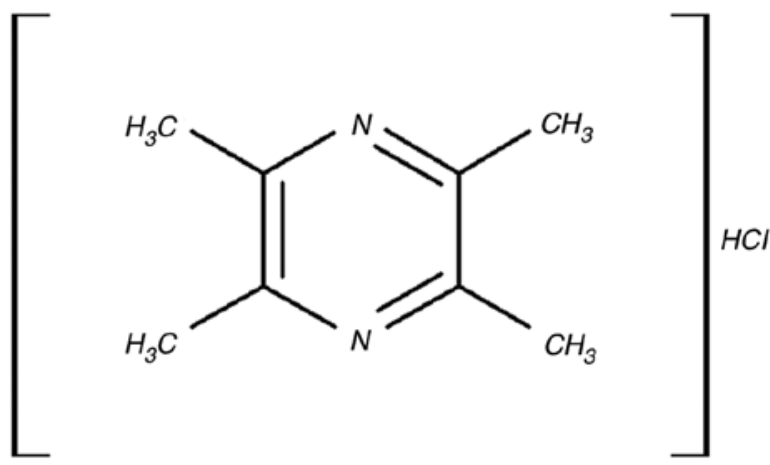

Chemical structure of ligustrazine hydrochloride (LH)

Figure 1. Chemical structure of ligustrazine and ligustrazine HCL. HCL, hydrochloride.

various concentrations of $\mathrm{LH}(0-3 \mathrm{mM})$ for $5 \mathrm{~min}$ at $37^{\circ} \mathrm{C}$. For the ex vivo experiment, an equal volume of PRP was collected from rats in each group and incubated in 5.5 $\mu \mathrm{M}$ ADP for $5 \mathrm{~min}$ at $37^{\circ} \mathrm{C}$. Following incubation, aggregation of platelets in each group was monitored by measuring light transmission via a platelet aggregometer (LBY-NJ4; Pulisheng Instrument Co., Ltd., Beijing, China), and the $\%$ maximum platelet aggregation was recorded.

Measurement of platelet intracellular $\mathrm{Ca}^{2+}$ mobilization. For the in vitro experiment, $1 \mathrm{ml}$ prepared $\mathrm{PRP}\left(3 \times 10^{8}\right.$ platelets $\left./ \mathrm{ml}\right)$ was pretreated with $\mathrm{LH}(0,1,2$ and $3 \mathrm{mM})$ in the presence of $\mathrm{CaCl}_{2}(1 \mathrm{mM})$ for $5 \mathrm{~min}$ at $37^{\circ} \mathrm{C}$. For the ex vivo experiment, $1 \mathrm{ml}$ prepared PRP $\left(3 \times 10^{8}\right.$ platelets $\left./ \mathrm{ml}\right)$ from the Control, Adrenaline and Adrenaline $+\mathrm{LH}$ rat groups were suspended in $\mathrm{CaCl}_{2}(1 \mathrm{mM})$ for $5 \mathrm{~min}$ at $37^{\circ} \mathrm{C}$. Subsequently, the platelets from in vitro and ex vivo incubations were stimulated with ADP $(5.5 \mu \mathrm{M})$ or IGF-1 $(300 \mu \mathrm{M})$ for $5 \mathrm{~min}$ at $37^{\circ} \mathrm{C}$. The platelets were incubated with Fluo-4/AM $(20 \mu \mathrm{M})$ for $60 \mathrm{~min}$ at $37^{\circ} \mathrm{C}$ in the dark and subsequently centrifuged at room temperature for $15 \mathrm{~min}$ at $500 \mathrm{x} \mathrm{g}$. The fluorescence intensity of 100,000 platelets/sample was examined using a flow cytometer (BD Biosciences, San Jose, CA, USA) and analyzed via BD CellQuest ${ }^{\mathrm{TM}}$ Pro (Version 6.0; BD Biosciences, San Jose, CA, USA).

Measurement of TXB2 expression levels. For the in vitro experiment, $1 \mathrm{ml}$ prepared PRP $\left(3 \times 10^{8}\right.$ platelets $\left./ \mathrm{ml}\right)$ was pretreated with $\mathrm{LH}(0,1,2$ and $3 \mathrm{mM})$ for $5 \mathrm{~min}$ at $37^{\circ} \mathrm{C}$. Subsequently, the platelets were stimulated with ADP $(5.5 \mu \mathrm{M})$ or IGF-1 $(300 \mu \mathrm{M})$ for $5 \mathrm{~min}$ at $37^{\circ} \mathrm{C}$. The PRP was centrifuged at $1,000 \times \mathrm{g}$ for $15 \mathrm{~min}$ at $4^{\circ} \mathrm{C}$. The supernatant was collected and TXB2 levels were determined using an ELISA kit (cat. no. ADI-900-002; Enzo Life Sciences, Inc.) and expressed as $\mathrm{pg} / \mathrm{ml}$. For the ex vivo experiment, blood was drawn from the Control, Adrenaline and Adrenaline $+\mathrm{LH}$ groups at the end of the experiment. Plasma was prepared by centrifuging for $15 \mathrm{~min}$ at $4^{\circ} \mathrm{C}$ at $1,600 \mathrm{x} \mathrm{g}$, and the levels of TXB2 in plasma were measured by ELISA, similarly to the in vitro experiment.

Tail bleeding assay. To evaluate the bleeding risk of $\mathrm{LH}$, a modified tail cutting method was used (30). Following adrenaline hydrochloride or saline injection for $1 \mathrm{~h}$, the rats in each group were anesthetized using sodium pentobarbital (45 mg/kg). Subsequently, the tail was pre-warmed for $3 \mathrm{~min}$ in saline solution at $37^{\circ} \mathrm{C}$. Bleeding was induced by precise transection of the mouse tail at $3 \mathrm{~mm}$ from the tip. The distal portion of the tail $(3 \mathrm{~cm})$ was immersed vertically into saline solution at $37^{\circ} \mathrm{C}$. The time between the start of transection to bleeding cessation was recorded as the bleeding time.

Western blot analysis. The expression levels of associated proteins in PRP from in vitro and ex vivo experiments were determined by western blot analysis. PRP from each group was lysed with mammalian cell radioimmunoprecipitation assay lysis buffer (cat. no. P0013; Beyotime Institute of Biotechnology; Haimen, China) containing protease and phosphatase inhibitor cocktails. Total protein concentrations were determined by a bicinchoninic acid assay. Equal amounts of total proteins $(50 \mu \mathrm{g})$ were resolved in $12 \%$ SDS-PAGE and electroblotted. The nitrocellulose membranes were blocked with $5 \%$ skimmed milk at room temperature for $2 \mathrm{~h}$ and incubated with primary antibodies targeting p-Akt (1:1,000; cat. no. 4060), Akt (1:1,000; cat no. 4685) or $\beta$-actin $(1: 1,000$; cat. no. 4967) overnight at $4^{\circ} \mathrm{C}$. Subsequently, the membranes were incubated with the appropriate HRP-conjugated anti-rabbit antibody (1:5,000; cat. no. 7074) at room temperature for $1 \mathrm{~h}$, followed by enhanced chemiluminescence detection (Thermo Fisher Scientific, Inc., Waltham, MA, USA). $\beta$-actin was used as a loading control.

Statistical analysis. All experiments were performed at least three times and presented as the mean \pm standard deviation. Statistical analyses were performed using SPSS for Windows (version 18.0; SPSS, Inc., Chicago, IL, USA). Analysis of three or more groups was performed by one-way analysis of variance, followed by the least-significant difference test. Values obtained in a number of experiments were converted into \% for comparison of controls with treated samples. $\mathrm{P}<0.05$ was considered to indicate a statistically significant difference.

\section{Results}

LH inhibits platelet aggregation in vitro and ex vivo. To examine the potential therapeutic effects of ligustrazine on 
A

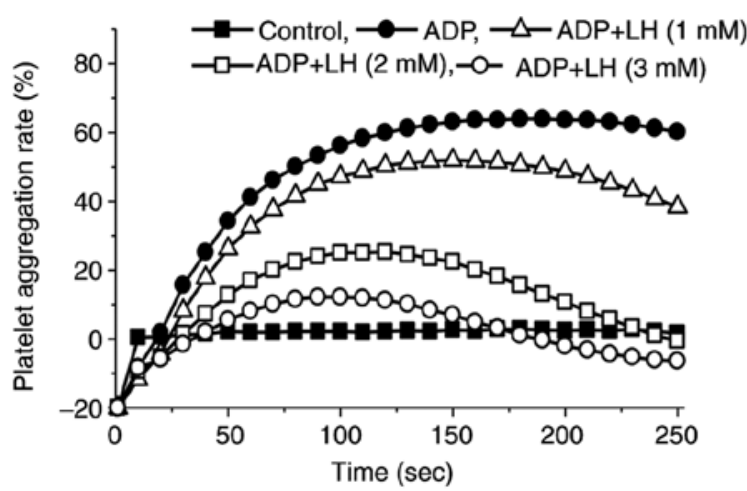

B

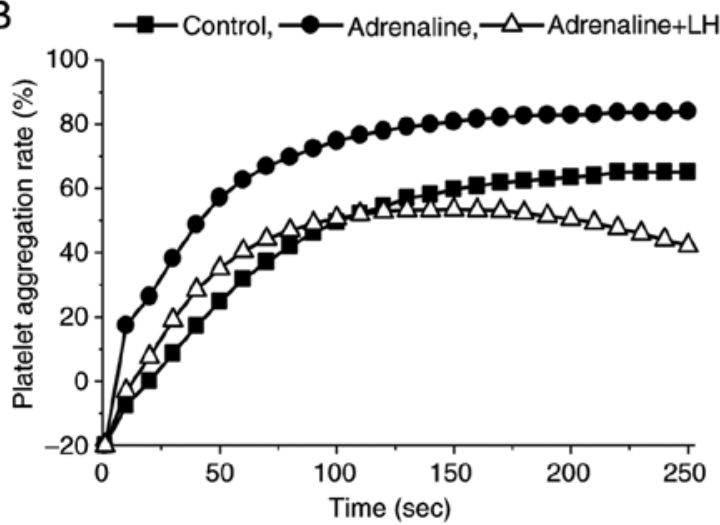

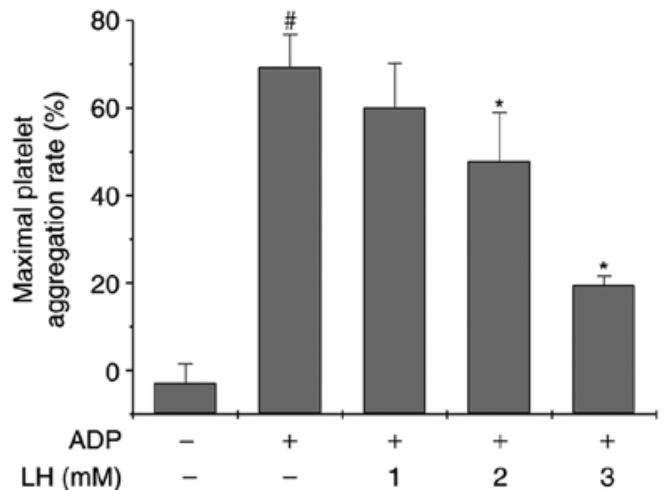

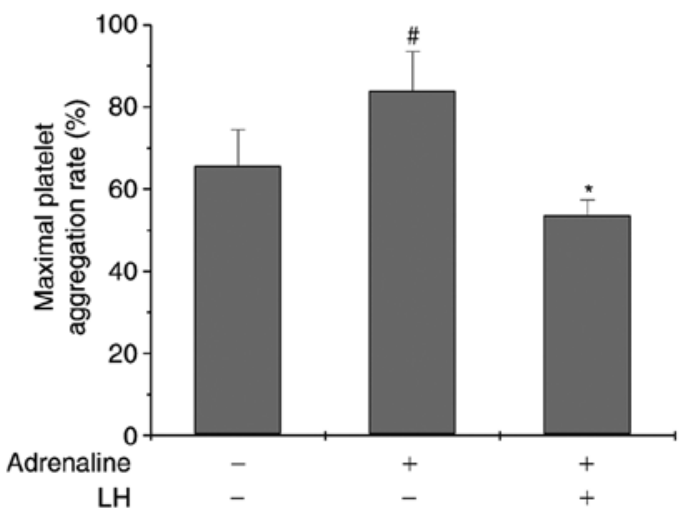

Figure 2. Effect of LH on ADP-induced platelet aggregation. (A) In vitro experiment. PRP was incubated with $5.5 \mu \mathrm{M}$ ADP following pretreatment with various concentrations of $\mathrm{LH}(0-3 \mathrm{mM})$. (B) Ex vivo experiment. PRP collected from rats in each group was stimulated with ADP (5.5 $\mu \mathrm{M})$. The platelet aggregation of PRP in vitro and ex vivo was determined by platelet aggregometer (left panel) and the \% maximum platelet aggregation was determined at 250 sec (right panel). Data are presented as mean \pm standard deviation from at least three repetitions. ${ }^{*} \mathrm{P}<0.05$ vs. untreated $\mathrm{PRP}$ or rats in Control group; ${ }^{*} \mathrm{P}<0.05$ vs. ADP-stimulated PRP or rats in the Adrenaline group. LH, ligustrazine hydrochloride; ADP, adenosine diphosphate; PRP, platelet-rich plasma.

platelet activation, rat PRP was incubated with LH, followed by stimulation with ADP. As illustrated in Fig. 2A, ADP stimulation markedly induced platelet aggregation in vitro, and this was significantly inhibited by treatment with $\mathrm{LH}$ in a dose-dependent manner ( $\mathrm{P}<0.05$ vs. untreated PRP; $\mathrm{P}<0.05$ vs. ADP-stimulated PRP). To verify the antiplatelet effect of LH, an ex vivo platelet activation model was employed, where the rats were pretreated with LH for 7 days followed by subcutaneous injection of adrenaline hydrochloride for $1 \mathrm{~h}$. Rat PRP from each group was collected and stimulated with ADP (used as stimulation for detection of platelet aggregation). As illustrated in Fig. 2B, treatment with LH significantly inhibited adrenaline-induced platelet aggregation $(\mathrm{P}<0.05$ vs. Control group; $\mathrm{P}<0.05$ vs. Adrenaline group). Taken together, these results suggested that ligustrazine possesses potent properties of suppressing platelet aggregation in vitro and ex vivo.

LH inhibits intracellular $\mathrm{Ca}^{2+}$ mobilization and TXA2 formation in vitro and ex vivo. To further determine the antiplatelet activity of $\mathrm{LH}$, its effect on $\mathrm{Ca}^{2+}$ mobilization and TXA2 secretion was investigated. As presented in Fig. 3A and C, LH significantly and dose-dependently inhibited ADP-induced $\mathrm{Ca}^{2+}$ mobilization and TXB2 secretion in platelets in vitro $(\mathrm{P}<0.05$ vs. untreated PRP; $\mathrm{P}<0.05$ vs. ADP-stimulated PRP). Furthermore, similar results were observed in the ex vivo platelet activation model (Fig. 3B and D; $\mathrm{P}<0.05$ vs. Control group; $\mathrm{P}<0.05$ vs. Adrenaline group), suggesting that the anti-platelet activity of ligustrazine may be mediated by the inhibition of $\mathrm{Ca}^{2+}$ mobilization and TXA2 formation.

LH suppresses phosphorylation of Akt in vitro and ex vivo. To examine the molecular mechanism of the antiplatelet effects of LH, its effect on the phosphorylation/activation of Akt was investigated. As presented in Fig. 4, ADP markedly increased the phosphorylation of Akt in vitro and ex vivo, compared with the control group; this effect was however significantly suppressed by treatment with LH. To further verify the inhibitory effect of LH on Akt signaling, a specific Akt pathway activator, IGF-1, was employed. The results in Fig. 5A demonstrated that LH treatment profoundly and dose-dependently suppressed IGF-1-induced Akt phosphorylation. The levels of total Akt were unaltered during the experiment. In addition, LH significantly inhibited IGF-1-induced platelet aggregation, $\mathrm{Ca}^{2+}$ mobilization and TXB2 secretion in platelets (Fig. 5B-D; $\mathrm{P}<0.05$ vs. untreated PRP; $\mathrm{P}<0.05$ vs. IGF-1-stimulated PRP). Collectively, these results suggested that ligustrazine exerts its inhibitory effects on platelet activation at least partly via suppression of the Akt signaling pathway.

LH displays low risk of hemorrhage in vivo. Multiple currently-used antithrombotic agents have adverse effects, including impaired blood coagulation or prolonged bleeding time. Therefore, the effect of LH on bleeding time was investigated in vivo in rats using a cutting tail method (30). As 
A

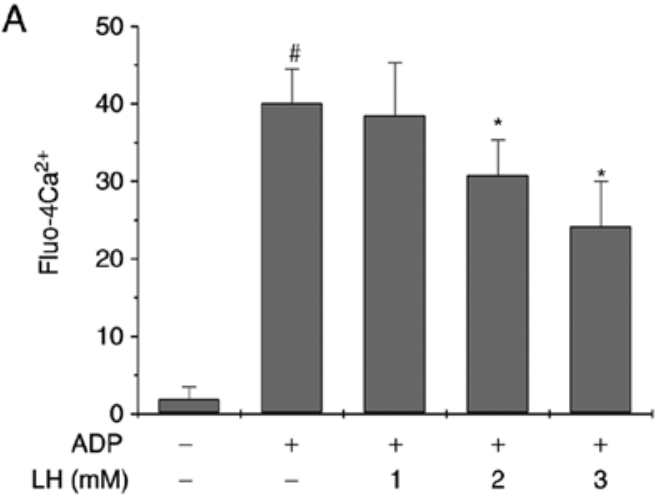

C

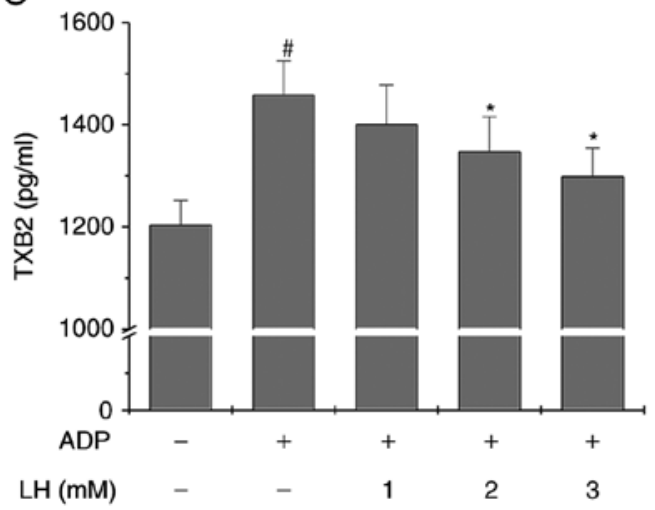

B

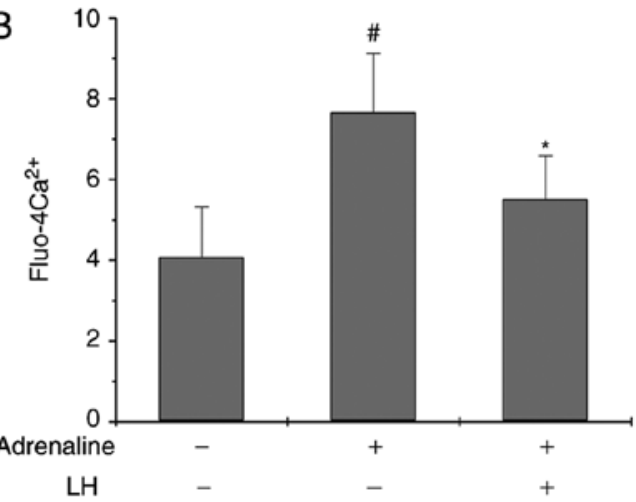

D

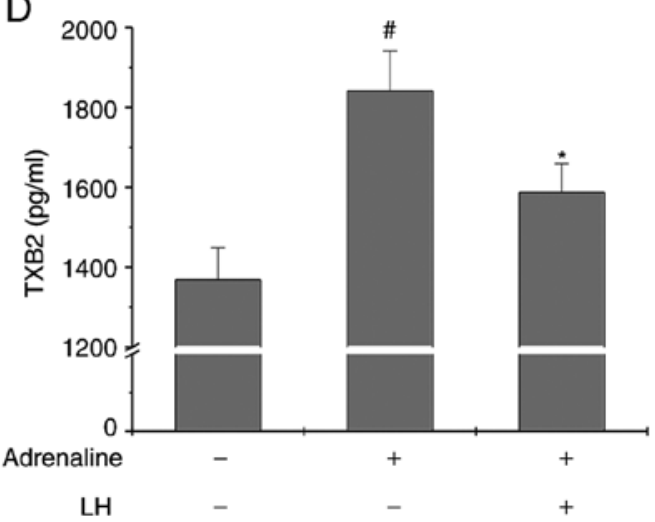

Figure 3. Effect of $\mathrm{LH}$ on intracellular $\mathrm{Ca}^{2+}$ mobilization and TXA2 formation. (A) PRP was pre-treated with various concentrations of $\mathrm{LH}$ (0-3 mM) in the presence of $\mathrm{CaCl}_{2}(1 \mathrm{mM})$. (B) PRP collected from rats in each group was suspended in $\mathrm{CaCl}_{2}(1 \mathrm{mM})$. The platelets from in vitro (A) and ex vivo (B) experiments were stimulated with ADP $(5.5 \mu \mathrm{M})$. The intracellular $\mathrm{Ca}^{2+}$ concentration in PRP was determined by Fluo-4/AM (20 $\left.\mu \mathrm{M}\right)$ staining, followed by flow cytometry analysis. (C) Supernatant was collected following pretreatment of PRP with various concentrations of LH (0-3 mM) followed by ADP (5.5 $\mu$ M) stimulation. (D) Plasma from rats in each group was collected. TXB2 levels in supernatants from the in vitro (C) and in plasma from the ex vivo (D) experiments were determined using an ELISA kit and expressed as $\mathrm{pg} / \mathrm{ml}$. Data are presented as mean \pm standard deviation from at least three repetitions. ${ }^{\#} \mathrm{P}<0.05$ vs. untreated PRP or rats in Control group; ${ }^{*} \mathrm{P}<0.05$ vs. ADP-stimulated PRP or rats in the Adrenaline group. LH, ligustrazine hydrochloride; TXA2, thromboxane A2; PRP, platelet-rich plasma; ADP, adenosine diphosphate; TXB2, thromboxane B2; Fluo-4/AM, fluo-4-acetoxymethyl ester.

A

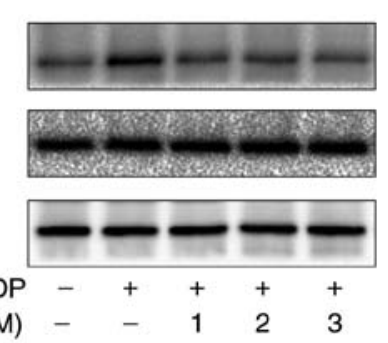

B

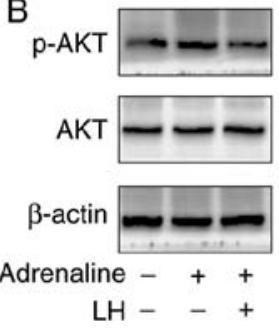

Figure 4. Effect of LH on phosphorylation of Akt. (A) PRP was incubated with $5.5 \mu \mathrm{M}$ ADP following pretreatment with various concentrations of $\mathrm{LH}$ (0-3 mM). (B) PRP collected from rats in each group was stimulated with ADP $(5.5 \mu \mathrm{M})$. The expression levels of Akt and p-Akt were determined by western blot analysis. $\beta$-actin was used as the internal control. Images are representative of three independent experiments. LH, ligustrazine hydrochloride; Akt, AKT serine/threonine kinase; PRP, platelet-rich plasma; ADP, adenosine diphosphate; p-, phosphorylated.

presented in Fig. 6, the bleeding time in the Adrenaline-treated group was shorter compared with the control group, while the bleeding time in the LH-pretreated group for 7 days was increased $(\mathrm{P}<0.05$ vs. Control group; $\mathrm{P}<0.05$ vs. Adrenaline group). However, LH did not significantly prolong the bleeding time compared with the control group, suggesting that ligustrazine may have low bleeding risk.

\section{Discussion}

Due to the critical role of hyper-activation of platelets in the development of thrombotic vascular diseases, the process of platelet activation has become an attractive therapeutic target. Numerous antiplatelet chemical drugs are used to prevent or treat thrombosis. However, the currently available drugs have specific clinical disadvantages, including gastrointestinal sideeffects and hemorrhage $(16,17)$. Therefore, there is an urgent requirement for safer and more effective antiplatelet agents without these adverse effects. In recent years, novel therapeutic agents have been derived from Chinese medicinal herbs and there is growing interest in this area. Ligustrazine, one of the natural alkaloids isolated from RLW that is commonly used to clinically treat cardiac-cerebral diseases, has been demonstrated to possess antiplatelet activity (19-22). However, the precise mechanism of its potential therapeutic effects remains to be further elucidated. Using in vitro and ex vivo platelet activation models, established by stimulating rat PRP with the platelet activator ADP, the present study demonstrated that $\mathrm{LH}$ (the clinical-grade form of ligustrazine) was able to significantly inhibit platelet aggregation. In addition, LH was able to reverse adrenaline-induced shortening of bleeding time, indicating the low bleeding risk of ligustrazine. However, the 
A

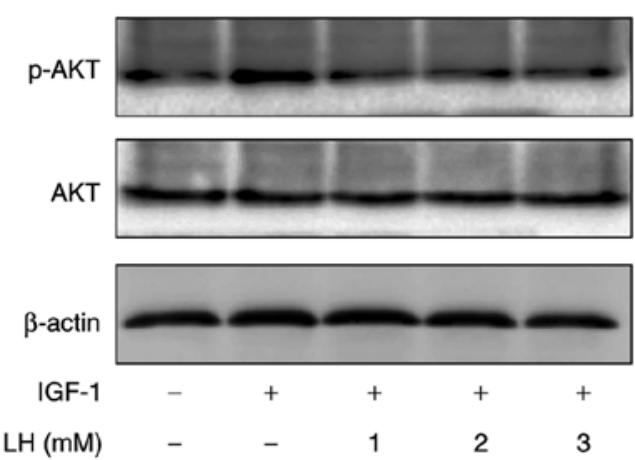

C

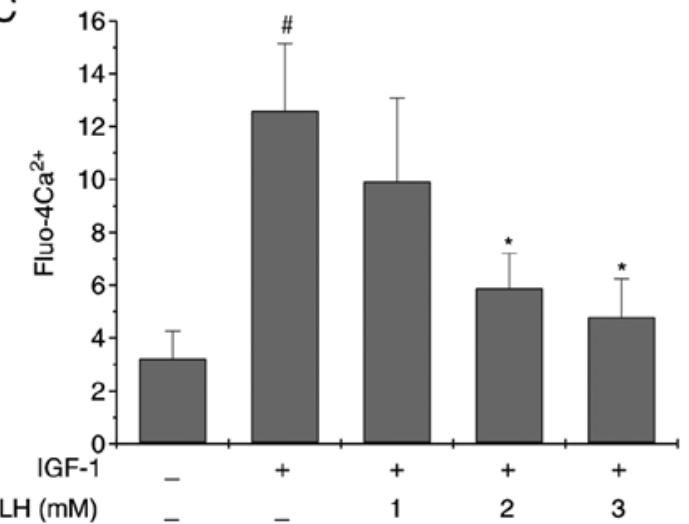

B
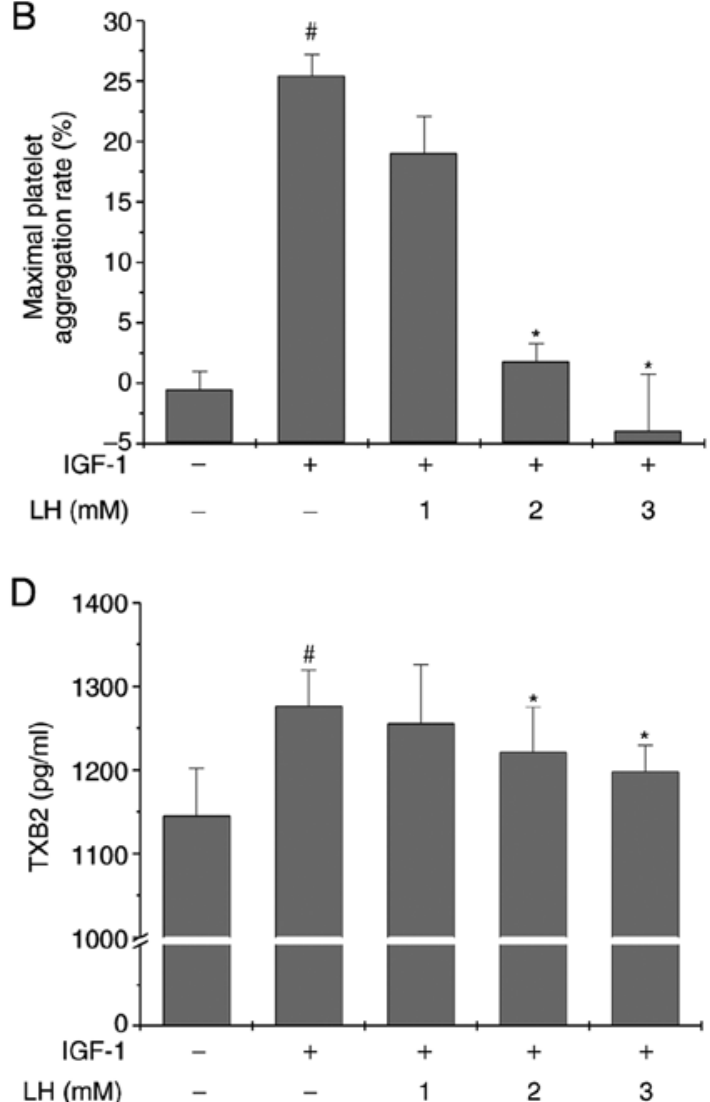

Figure 5. Effect of LH on IGF-1-induced activation of the Akt signaling pathway. PRP were stimulated with IGF-1 (300 $\mu$ M), following pretreatment with various concentrations of LH (0-3 mM). (A) Protein expression levels of Akt and p-Akt in PRP were determined by western blot analysis. $\beta$-actin was used as the internal control. (B) Platelet aggregation was measured using a platelet aggregometer and the \% of maximum platelet aggregation was determined at $250 \mathrm{sec}$. (C) The intracellular $\mathrm{Ca}^{2+}$ concentration in PRP was determined by Fluo-4/AM (20 $\mu$ M) staining, followed by flow cytometry analysis. (D) Levels of TXB2 in the supernatants of PRP was determined by ELISA and expressed as pg/ml. Experiments were performed at least three independent times. "P $<0.05$ vs. untreated PRP; * P<0.05 vs. IGF-1-stimulated PRP. LH, ligustrazine hydrochloride; IGF-1, insulin-like growth factor-1; Akt, AKT serine/threonine kinase; PRP, platelet-rich plasma; TXB2, thromboxane B2; p, phosphorylated; Fluo-4/AM, fluo-4-acetoxymethyl ester.

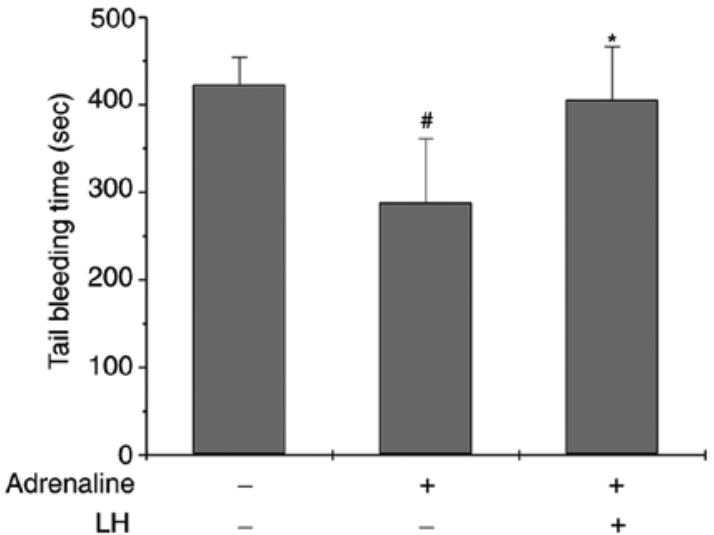

Figure 6. Effect of LH on bleeding time. Following administration of LH or saline for 7 days, the rats were injected with adrenaline hydrochloride or saline for $1 \mathrm{~h}$. Bleeding risk was evaluated using a modified tail cutting method. The accumulated bleeding time was recorded within $600 \mathrm{sec} . \mathrm{n}=5$ rats from each group were used in this experiment. ${ }^{\#} \mathrm{P}<0.05$ vs. Control group; ${ }^{*} \mathrm{P}<0.05$ vs. Adrenaline group. LH, ligustrazine hydrochloride.

rapid metabolism and short half-life of ligustrazine severely limits its clinical application. Therefore, novel chemical forms of ligustrazine with a longer half-life require development in further studies. Additionally, a comparison of LH with other antiplatelet medicines (including aspirin and clopidelgrel) and their potential combinations require further assessment.

Platelet activation may be induced by a variety of agonists, including ADP and TXA2, which are released from the granules of activated platelets. TXA2 exerts its function through interaction with the thromboxane receptor, contributing to the amplification of the initial platelet activation, which therefore is a principal target for most currently-used antiplatelet agents (31-33). Although different agonists induce platelet activation through different mechanisms by respectively binding to their specific receptors, they all result in an elevation of intracellular $\mathrm{Ca}^{2+}$ concentration $\left[\left(\mathrm{Ca}^{2+}\right) \mathrm{i}\right]$, an event termed $\mathrm{Ca}^{2+}$ mobilization (34). $\mathrm{Ca}^{2+}$ is an important second messenger that is critical for various cellular processes, including platelet activation (35). Therefore, the present study verified the antiplatelet activity of $\mathrm{LH}$ by examining $\mathrm{Ca}^{2+}$ mobilization and TXA2 secretion, and the results demonstrated that $\mathrm{LH}$ significantly and dose-dependently inhibited ADP-induced $\mathrm{Ca}^{2+}$ mobilization and TXB2 secretion in platelets in vitro and ex vivo. These results suggested that the antiplatelet activity of ligustrazine may be mediated by the inhibition of $\mathrm{Ca}^{2+}$ mobilization and TXA2 formation.

It is well documented that the process of platelet activation is highly regulated by multiple pathways, including the PI3K/Akt 
signaling pathway. Activation of the PI3K/Akt pathway results in granule secretion and the second wave of platelet aggregation (9,36-40). To examine the mechanism of the antiplatelet activities of ligustrazine, its effect on the phosphorylation/activation of Akt was determined. The present study demonstrated that treatment with LH significantly suppressed ADP-induced Akt phosphorylation in vitro and ex vivo. Notably, using the specific Akt pathway activator IGF-1, the present study further confirmed that LH significantly and specifically suppressed the activation of Akt signaling. Consequently, treatment with LH markedly inhibited IGF-1-induced platelet aggregation, $\mathrm{Ca}^{2+}$ mobilization and TXB2 secretion in platelets. However, as phosphorylation of Akt is regulated by upstream regulators (including PI3K, PH domain and leucine rich repeat protein phosphatase 1, phosphatase and tensin homolog and associated microRNAs) (41-43) and exerts its function by regulating the expression of downstream molecules (including mammalian target of rapamycin) (44), the effect of LH upstream and downstream of Akt requires further assessment.

In conclusion, the present study proposed that ligustrazine possesses a broad range of antiplatelet activities, without apparent hemorrhagic side-effects. Suppression of Akt signaling may be one of the mechanisms by which ligustrazine exerts its antiplatelet function. The present results provide further preliminary evidence to support that ligustrazine may be considered a potent therapeutic agent against thrombotic vascular diseases.

\section{Acknowledgements}

Not applicable.

\section{Funding}

The present study was sponsored by the Natural Science Foundation of Fujian Province (grant no. 2018J01229) and the National Natural Science Foundation of China (grant no. 81774135).

\section{Availability of data and materials}

The analyzed datasets generated during the study are available from the corresponding author on reasonable request.

\section{Authors' contributions}

$\mathrm{KC}$ and JP conceived and designed the experiments. LL, AS and QL conducted the animal experiments. HC and JC performed FACS analysis. LL, YC and LYL performed the western blotting, ELISA and data analysis. HC and AS determined platelet aggregation analysis. LL, JP and $\mathrm{KC}$ wrote and revised the manuscript. All authors read and approved the final manuscript.

\section{Ethics approval and consent to participate}

All experiments involving animals were approved by the Institutional Animal Care and Use Committee of Fujian University of Traditional Chinese Medicine (Fuzhou, China).

\section{Patient consent for publication}

Not applicable.

\section{Competing interests}

The authors declare that they have no competing interests.

\section{References}

1. Smith T, Dhunnoo G, Mohan I and Charlton-Menys V: A pilot study showing an association between platelet hyperactivity and the severity of peripheral arterial disease. Platelets 18: 245-248, 2007.

2. Georgescu A, Alexandru N, Andrei E, Dragan E, Cochior D and Dias S: Effects of transplanted circulating endothelial progenitor cells and platelet microparticles in atherosclerosis development. Biol Cell 108: 219-243, 2016.

3. Alexandru N, Popov D and Georgescu A: Platelet dysfunction in vascular pathologies and how can it be treated. Thromb Res 129: 116-126, 2012.

4. Sharma G and Berger JS: Platelet activity and cardiovascular risk in apparently healthy individuals: A review of the data. J Thromb Thrombolysis 32: 201-208, 2011.

5. Angiolillo DJ: The evolution of antiplatelet therapy in the treatment of acute coronary syndromes: From aspirin to the present day. Drugs 72: 2087-2116, 2012.

6. Spiel AO, Gilbert JC and Jilma B: von Willebrand factor in cardiovascular disease: Focus on acute coronary syndromes. Circulation 117: 1449-1459, 2008.

7. Brass LF, Manning DR, Cichowski K and Abrams CS: Signaling through $\mathrm{G}$ proteins in platelets: To the integrins and beyond. Thromb Haemost 78: 581-589, 1997.

8. Franke TF, Kaplan DR, Cantley LC and Toker A: Direct regulation of the Akt proto-oncogene product by phosphatidylinositol-3,4-bisphosphate. Science 275: 665-668, 1997.

9. Guidetti GF, Canobbio I and Torti M: PI3K/Akt in platelet integrin signaling and implications in thrombosis. Adv Biol Regul 59: 36-52, 2015.

10. Moore SF, van den Bosch MT, Hunter RW, Sakamoto K, Poole AW and Hers I: Dual regulation of glycogen synthase kinase $3(\mathrm{GSK} 3) \alpha / \beta$ by protein kinase $\mathrm{C}(\mathrm{PKC}) \alpha$ and Akt promotes thrombin-mediated integrin $\alpha_{15} \beta_{3}$ activation and granule secretion in platelets. J Biol Chem 288: 3918-3928, 2013.

11. Chen X, Zhang Y, Wang Y, Li D, Zhang L, Wang K, Luo X, Yang Z, Wu Y and Liu J: PDK1 regulates platelet activation and arterial thrombosis. Blood 121: 3718-3726, 2013.

12. Yin H, Stojanovic A, Hay N and Du X: The role of Akt in the signaling pathway of the glycoprotein Ib-IX induced platelet activation. Blood 111: 658-665, 2008.

13. Kim S, Mangin P, Dangelmaier C, Lillian R, Jackson SP, Daniel JL and Kunapuli SP: Role of phosphoinositide 3-kinase beta in glycoprotein VI-mediated Akt activation in platelets. J Biol Chem 284: 33763-33772, 2009.

14. Reséndiz JC, Kroll MH and Lassila R: Protease-activated receptor-induced Akt activation-regulation and possible function. J Thromb Haemost 5: 2484-2493, 2007.

15. Chen J, De S, Damron DS, Chen WS, Hay N and Byzova TV: Impaired platelet responses to thrombin and collagen in AKT-1-deficient mice. Blood 104: 1703-1710, 2004.

16. Di Minno MN, Lupoli R, Palmieri NM, Russolillo A, Buonauro A and Di Minno G: Aspirin resistance, platelet turnover, and diabetic angiopathy: A 2011 update. Thromb Res 129: 341-344, 2012.

17. Uchiyama S: Clopidogrel resistance: Identifying and overcoming a barrier to effective antiplatelet treatment. Cardiovasc Ther 29: e100-e111, 2011.

18. Beijing Institute of Pharmaceutical Industry: Studies of active components of Ligusticum Wallichii Franch. I. Extraction, isolation and structure identification of tetramethylpyrazine. Chin Med J 7: 420-421, 1977 (In Chinese).

19. Li Z, Li D, Huang J, Zhang W, Ding Y and Wang S: Preparation of cardiovascular disease-related genes microarray and its application in exploring ligustrazine-induced changes in endothelial gene expression. Pol J Pharmacol 56: 427-433, 2004. 
20. Zhang F, Ni C, Kong D, Zhang X, Zhu X, Chen L, Lu Y and Zheng S: Ligustrazine attenuates oxidative stress-induced activation of hepatic stellate cells by interrupting platelet-derived growth factor- $\beta$ receptor-mediated ERK and p38 pathways. Toxicol Appl Pharmacol 265: 51-60, 2012.

21. Li S, Chen H, Wang X, Wu J, Jiang J and Wang Y: Pharmacokinetic study of a novel stroke therapeutic, 2-[[(1,1-dimethylethyl)oxidoimino]methyl]-3,5,6-trimethylpyrazine, by a simple HPLC-UV method in rats. Eur J Drug Metab Pharmacokinet 36 95-101, 2011.

22. Wu W, Yu X, Luo XP, Yang SH and Zheng D: Tetramethylpyrazine protects against scopolamine-induced memory impairments in rats by reversing the $\mathrm{cAMP} / \mathrm{PKA} / \mathrm{CREB}$ pathway. Behav Brain Res 253: 212-216, 2013.

23. Cheng XR, Zhang L, Hu JJ, Sun L and Du GH: Neuroprotective effects of tetramethylpyrazine on hydrogen peroxide-induced apoptosis in PC12 cells. Cell Biol Int 31: 438-443, 2007.

24. Han J, Song J, Li X, Zhu M, Guo W, Xing W, Zhao R, He X, Liu X, Wang S, et al: Ligustrazine suppresses the growth of HRPC cells through the inhibition of cap-dependent translation via both the mTOR and the MEK/ERK pathways. Anticancer Agents Med Chem 15: 764-772, 2015.

25. Wang GJ: Changes of nail fold microcirculation in patients with acute cerebral thrombosis treated with ligustrazine. Chin J Neurol Psychiatry 17: 121-124, 1984 (In Chinese).

26. Sheu JR, Kan YC, Hung WC, Ko WC and Yen MH: Mechanisms involved in the antiplatelet activity of tetramethylpyrazine in human platelets. Thromb Res 88: 259-270, 1997.

27. Lin CI, Wu SL, Tao PL, Chen HM and Wei J: The role of cyclic AMP and phosphodiesterase activity in the mechanism of action of tetramethylpyrazine on human and dog cardiac and dog coronary arterial tissues. J Pharm Pharmacol 45: 963-966, 1993.

28. Liu SF, Cai YN, Evans TW, McCormack DG, Barer GR and Barnes PJ: Ligustrazine is a vasodilator of human pulmonary and bronchial arteries. Eur J Pharmacol 191: 345-350, 1990.

29. Peng W, Hucks D, Priest RM, Kan YM and Ward JP: Ligustrazineinduced endothelium-dependent relaxation in pulmonary arteries via an NO-mediated and exogenous L-arginine-dependent mechanism. Br J Pharmacol 119: 1063-1071, 1996

30. Alshbool FZ, Karim ZA, Vemana HP, Conlon C, Lin OA and Khasawneh FT: The regulator of G-protein signaling 18 regulates platelet aggregation, hemostasis and thrombosis. Biochem Biophys Res Commun 462: 378-382, 2015.

31. Su XL, Su W, Wang Y, Wang YH, Ming X and Kong Y: The pyrrolidinoindoline alkaloid Psm2 inhibits platelet aggregation and thrombus formation by affecting PI3K/Akt signaling. Acta Pharmacol Sin 37: 1208-1217, 2016.

32. Sakariassen KS, Alberts P, Fontana P, Mann J, Bounameaux H and Sorensen AS: Effect of pharmaceutical interventions targeting thromboxane receptors and thromboxane synthase in cardiovascular and renal diseases. Future Cardiol 5: 479-493, 2009.
33. Fontana P, Zufferey A, Daali Y and Reny JL: Antiplatelet therapy: Targeting the TxA ${ }_{2}$ pathway. J Cardiovasc Transl Res 7: 29-38, 2014

34. Varga-Szabo D, Braun A and Nieswandt B: Calcium signaling in platelets. J Thromb Haemost 7: 1057-1066, 2009.

35. Berridge MJ, Bootman MD and Roderick HL: Calcium signalling: Dynamics, homeostasis and remodelling. Nat Rev Mol Cell Biol 4: 517-529, 2003

36. Heraud JM, Racaud-Sultan C, Gironcel D, Albigès-Rizo C, Giacomini T, Roques S, Martel V, Breton-Douillon M, Perret B and Chap $\mathrm{H}$ : Lipid products of phosphoinositide 3-kinase and phosphatidylinositol 4',5'-bisphosphate are both required for ADP-dependent platelet spreading. J Biol Chem 273: 17817-17823, 1998

37. Canobbio I, Stefanini L, Cipolla L, Ciraolo E, Gruppi C, Balduini C, Hirsch E and Torti M: Genetic evidence for a predominant role of PI3Kbeta catalytic activity in ITAM- and integrin-mediated signaling in platelets. Blood 114: 2193-2196, 2009.

38. Woulfe DS: Akt signaling in platelets and thrombosis. Expert Rev Hematol 3: 81-91, 2010.

39. O'Brien KA, Stojanovic-Terpo A, Hay N and Du X: An important role for Akt3 in platelet activation and thrombosis. Blood 118: 4215-4223, 2011.

40. Stojanovic A, Marjanovic JA, Brovkovych VM, Peng X, Hay N, Skidgel RA and Du X: A phosphoinositide 3-kinase-AKT-nitric oxide-cGMP signaling pathway in stimulating platelet secretion and aggregation. J Biol Chem 281: 16333-16339, 2006.

41. Laurent PA, Severin S, Gratacap MP and Payrastre B: Class I PI 3-kinases signaling in platelet activation and thrombosis: PDK1/Akt/GSK3 axis and impact of PTEN and SHIP1. Adv Biol Regul 54: 162-174, 2014.

42. Meuillet EJ: Novel inhibitors of AKT: Assessment of a different approach targeting the pleckstrin homology domain. Curr Med Chem 18: 2727-2742, 2011.

43. Ishibashi O, Akagi I, Ogawa Y and Inui T: MiR-141-3p is upregulated in esophageal squamous cell carcinoma and targets pleckstrin homology domain leucine-rich repeat protein phosphatase-2, a negative regulator of the PI3K/AKT pathway. Biochem Biophys Res Commun 501: 507-513, 2018.

44. Razmara M, Heldin CH and Lennartsson J: Platelet-derived growth factor-induced Akt phosphorylation requires mTOR/Rictor and phospholipase C- $\gamma 1$, whereas S6 phosphorylation depends on mTOR/Raptor and phospholipase D. Cell Commun Signal 11: 3, 2013. 